\title{
22
}

\section{A Novel Dynamic Priority Scheduling Method For Multiple Classes Of ATM Traffic In An ATM Statistical Multiplexer}

\author{
P.A.Ganos ${ }^{a}$, M.N.Koukias ${ }^{a}$, G.K.Kokkinakis ${ }^{a}$ and S.A.Kotsopoulos ${ }^{b}$ \\ "Wire Communications Laboratory, Department of Electrical Engineering, \\ University of Patras, Patras, Greece \\ 'Wireless Communications Laboratory, Department of Electrical Engineering, \\ University of Patras, Patras, Greece
}

\begin{abstract}
The purpose of this paper is to propose a new priority scheduling method that dynamically manages four classes of ATM traffic and assigns different priority levels according to the QoS requirements of each class. Its performance has been studied by simulation under the application on an ATM statistical multiplexer. The results have shown that this scheme satisfies the loss and delay requirements of the ATM classes for a large range of traffic load.
\end{abstract}

Keyword Codes: H.4.3; H.5.1; I.6.3

Keywords: Information systems applications, Communications applications; Information interfaces and presentations, Multimedia information systems; Simulation and modeling, Applications.

\section{INTRODUCTION}

Asynchronous Transfer Mode (ATM) is the standard transport and switching technique adopted by CCITT [1] for the implementation of Broadband Integrated Services Digital Networks (B-ISDN) and provides high flexibility of network access, dynamic bandwidth allocation on demand and flexible bearer capacity allocation. ATM networks process all information types (continuous data streams or variable length packets) in single-size cells. Thus voice, video and bulk data services share ATM network resources on a dynamic basis. Although services use standard ATM cells, they require different and specific standards of service for their payloads [2,3]. For instance, real-time services such as voice have strict delay requirements, while data applications are characterized by high sensitivity in loss and video tends to be affected by cell loss and cell delay. Also, within delay sensitive traffic (e.g., voice or video), different traffic streams may contain more urgent information than others. 
The traffic sources in an ATM network are bursty and may generate cells at a near-peak rate during their active period. In order to make use of the ATM network resources more efficiently and support multiple classes of traffic, while satisfying their Quality of Service $(\mathrm{Q} 0 \mathrm{~S})$ requirements such as delay time and loss probability, priority traffic control mechanisms are required. According to these mechanisms ATM traffic sources are classified into multiple traffic classes and different priority levels are introduced to meet the QoS requirements of each class.

Several priority control schemes have been recently proposed for providing throughput and delay guarantees of the buffered cells at a switching node of an ATM network. A priority scheme takes action as a scheduling method (queueing discipline) of the buffered ATM cells. In most schemes two classes of traffic are considered: delay sensitive traffic and loss sensitive traffic. The simplest priority scheme is the Fixed priority scheme [4]. According to this scheme, priority is always given to the higher priority class (delay sensitive) and this class is always scheduled for service before the lower priority class (loss sensitive). The lower priority class is scheduled only in the case that there are not any buffered cells of the higher priority class. This scheme causes relatively high losses for the lower priority class while providing relatively low delays for the higher priority class. If the higher priority class occupies a large portion of the network traffic, the performance of the lower priority class will be severely degraded.

The Fixed priority scheme is characterized as a static one, since priority is always given to one traffic class. In an ATM environment the dynamic priority schemes are more flexible because the priority level changes with time taking into account the loss and delay requirements of each class. Two well known dynamic priority schemes are the Minimum Laxity Threshold (MLT) [5] and the Queue Length Threshold (QLT) [5], which assign different priority levels to two classes of traffic (delay sensitive and loss sensitive) in each time-slot. In the MLT scheme, each delay sensitive cell is characterized by a laxity which is the time until the expiration of the cell's deadline. Upon the arrival of a delay sensitive cell, its laxity is equal to its deadline and decreases by one in each time-slot. A delay sensitive cell remains in its buffer until it is either transmitted or its laxity reaches zero. In the latter case the cell is discarded and considered lost. In the MLT scheme, priority is given to the delay sensitive class cells, if there are any delay sensitive buffered cells, whose laxity is less than a threshold value; otherwise priority is given to the loss sensitive traffic cells. In the QLT scheme, priority is given to the loss sensitive traffic cells, when the number of the buffered loss sensitive cells exceeds a threshold value; otherwise priority is given to the delay sensitive buffered cells.

As mentioned above, even within delay sensitive traffic, there may be different classes with different delay requirements. Head-of-the-Line with Priority Jumps (HOL-PJ) [6], Delay Earliest Due Date (Delay-EDD) [7] and Balancing Discipline (BD) [8], are three representative examples of dynamic priority schemes, which satisfy the different delay requirements of two delay sensitive classes. In these schemes, higher priority is given to the delay sensitive class with the stricter delay requirements. 
Although the schemes mentioned earlier assign priorities to the various classes of ATM traffic in a dynamic manner, improving the network performance over the fixed-priority discipline, they have been studied for only two ATM traffic classes with general QoS requirements. In [9] a dynamic priority queueing scheme was presented, which assigns transmission priorities to three ATM classes, one loss sensitive and two delay sensitive. Although this scheme manages efficiently the QoS requirements of the three classes, the traffic models used in simulation do not represent any specific ATM service classes. In an ATM environment, multiple classes of traffic are provided and each ATM class has specific performance requirements. So, an appropriate priority scheme is necessary, which must satisfy the specific cell loss requirements (zero or controllable loss) and the specific cell delay requirements (bounded or unbounded delay and jitter) of multiple ATM traffic classes.

In this paper a novel dynamic priority control scheme is proposed, called Four Classes Priority Scheme (FCPS). The performance of the FCPS has been studied by simulation under its application on a $16 \times 4$ Multiple-Input MultipleOutput (MIMO) statistical multiplexer. Four ATM classes with different source models are considered: a Loss and Delay Sensitive Class (LDSC, e.g. video), a Loss Sensitive Class (LSC, e.g. critical data), and two Delay Sensitive Classes (DSC) with different delay requirements (e.g. voice and regular real-time data with some tolerance in loss). The cells of each class are buffered in separate output queues. The FCPS uses two priority methods: a space priority method and a service priority method. The space priority method is used for the control of the buffer space when an overflow condition (LDSC, LSC and DSC) occurs and/or when the expiration of the maximum admissible delay of the delay sensitive cells (LDSC and DSC) takes place. The service priority method is used as a scheduling method and assigns transmission priorities to the buffered cells. The proposed priority scheme is characterized by high flexibility, i.e. various parameters affect the tradeoffs between the cell loss probability and the cell delay of the four classes. The performance results obtained by simulation show that this scheme fulfills the QoS requirements of each class for a large range of traffic load.

This paper is organized as follows: In section 2, the structure of a $16 \times 4$ MIMO statistical multiplexer is described. In section 3, the traffic models of the four ATM service classes are presented in detail. In section 4, a description of the proposed priority control scheme is given. In section 5 , a simulation model for the evaluation of the priority scheme is described. In section 6 , the simulation results are discussed. Finally, in section 7, concluding remarks are provided.

\section{A 16x4 MIMO STATISTICAL MULTIPLEXER}

In order to evaluate the performance of the proposed FCPS, we applied this scheme to a 16X4 Multiple-Input Multiple-Output (MIMO) statistical multiplexer with output buffering. In [10] it has been shown that this kind of multiplexer appears less average cell delay and smaller cell loss probability due to buffer overflow, than the multiple-input single-output multiplexer. The concept of multiple outputs (channel grouping) has also been applied to [11] to 
improve the ATM switch's performance in terms of throughput, cell delay and cell loss probability.

In our model, each input link of the MIMO multiplexer carries cells from all priority classes. The cells generated from Svv video sources, Svo voice sources, $S_{\mathrm{CD}}$ critical data sources and $S_{\mathrm{RD}}$ regular real-time data sources are first multiplexed via a bufferless multiplexer to a higher speed transmission link (e.g. STS-3c channel). Since no buffers are used, this kind of multiplexer does not cause any delay to the incoming cells. Also, if the source model of each traffic type is known (Section 3), then the mean total load that $S_{i}$ sources ( $i=V D, V O$, $\mathrm{CD}, \mathrm{RD}$ ) contribute to the multiplexer inputs can be calculated and cell losses in the bufferless multiplexer can be avoided. Sixteen multiplexers are used and their outputs are fed to the inputs of the MIMO multiplexer. Hence a total of $16 \mathrm{x} \mathrm{S}_{\mathrm{VD}}$ video sources, $16 \mathrm{x} \mathrm{S}_{\mathrm{vo}}$ voice sources, $16 \mathrm{xS}_{\mathrm{CD}}$ critical data sources and $16 \mathrm{xS}$ RD regular real-time data sources contribute a mixed load to the MIMO multiplexer inputs.

The MIMO statistical multiplexer consists of four components (Figure 1): a Time Division Multiplexer (TDM), a Cell Distributor, an Output Buffer Module and an Arbiter. The cells coming into the 16 inputs are time division multiplexed by the TDM into a higher-speed channel, which is fed to the Cell Distributor. The Cell Distributor reads the VPI/VCI value of an incoming cell to identify its priority level (service class of each cell) and distributes it to the appropriate buffer. The priority level can be registered in a look-up table which is initialized by the connection admission control at connection setup time and contains the values of all necessary connection traffic parameters. The Output Buffer Module consists of four buffers one for each class. The use of output queues has been proven to give the best delay/throughput performance [12]. The Arbiter chooses four cells (equal to the number of the MIMO multiplexer outputs) according to the FCPS and transmits them to the four output lines of the multiplexer.

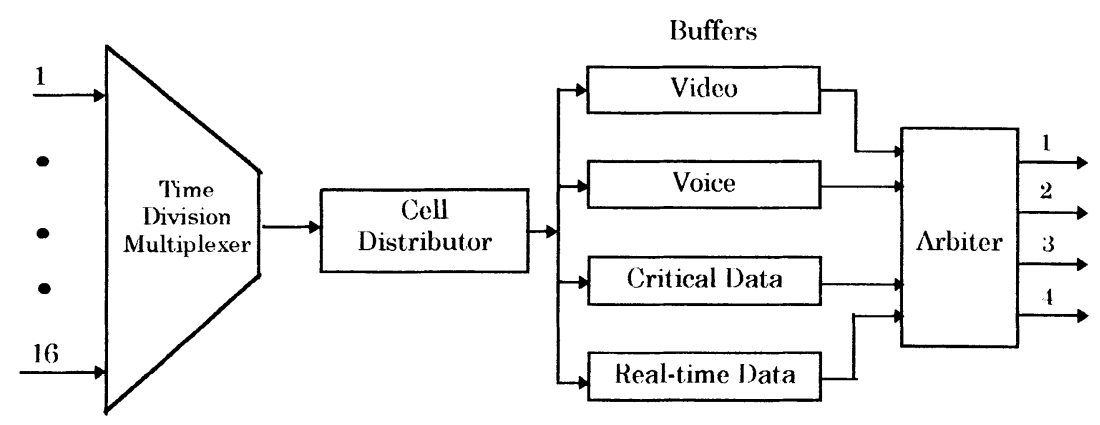

Figure 1: 16x4 MIMO statistical multiplexer

Since the buffered cells are transmitted to four channels simultaneously, it may be guaranteed that all channels in the same group will be carried on a single physical transmission link so that all channels will experience the same 
amount of propagation delay. This may be achieved if four STS-3c channels (155.52 Mbit/s) are byte-interleaved into a STM-4 (622.08 Mbit/s) channel and transmitted through a fiber trunk to the next node.

\section{DESCRIPTION OF THE TRAFFIC MODELS OF ATM CLASSES}

The proposed FCPS supports four ATM classes: a Loss and Delay Sensitive Class (LDSC, e.g. video), a Loss Sensitive Class (LSC, e.g. critical data), and two Delay Sensitive Classes (DSC) with different delay requirements (e.g. voice and regular real-time data with tolerance in loss). Different source models are considered for each type of traffic, which are described in the following.

\subsection{TRAFFIC MODEL FOR VIDEO SOURCES}

Video sources are usually compressed by using an interframe variable-rate coding scheme which encodes only significant differences between successive frames. Video source generate correlated cell arrivals and the traffic originated from these sources is considered to be very bursty. In our work, we use a model for video sources, which was presented in [13]. Note that, this model is used for video sources without scene changes and considers only short-term correlation which corresponds to uniform activity levels. According to this model, video sources are approximated by a discrete state, continuous - time Markov process.

The process $\lambda_{N}(t)$ describing the bit rate of $N$ video sources at time $t$ is approximated by a continuous - time process $\bar{\lambda}_{N}(\dagger)$ with discrete jumps at random Poisson times. The state transition diagram $\bar{\lambda}_{N}(t)$ is shown in Figure 2.

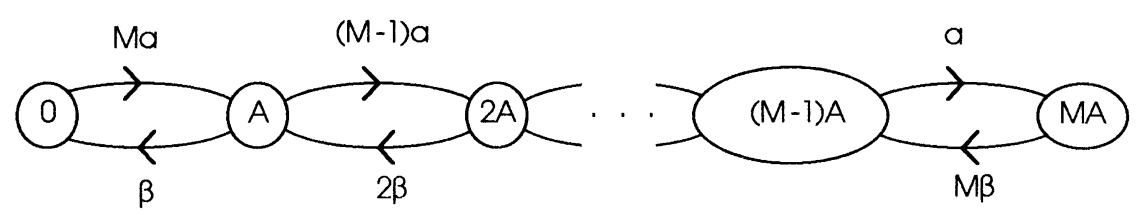

Figure 2: State transition diagram

The aggregated arrival process from $\mathrm{N}$ video sources makes transitions between $\mathrm{M}+1$ levels $(0, \mathrm{~A}, \ldots, \mathrm{MA})$, assuming a constant uniform quantization step A bits/pixel. The label in each state indicates the data rate in that state. The $\bar{\lambda}_{N}(\dagger)$ at steady state will have a binomial distribution with mean $E\left(\bar{\lambda}_{N}\right)$, variance $\overline{\mathrm{C}}_{N}(0)$ and exponential autocovariance $\overline{\mathrm{C}}_{N}(\mathrm{~T})$, given by the following equations: 


$$
\begin{aligned}
& P\left(\bar{\lambda}_{N}(t)=k A\right\}=\frac{M !}{k !(M-k) !} p^{k}(1-p)^{M-k} \\
& P=\frac{a}{a+\beta} \\
& E\left(\bar{\lambda}_{N}\right)=M A P \\
& \bar{C}_{N}(0)=M^{2} p(1-p) \\
& \bar{C}_{N}(T)=\bar{C}_{N}(0) e^{-(a+\beta) T}
\end{aligned}
$$

Given $M$ and $N$, the parameters $\beta$, $a$ and $A$ are matched with the measured data in [13] and are given by the following equations:

$$
\beta=\frac{a}{1+\frac{N \times E^{2}\left(\lambda_{N}\right)}{M \times C_{N}(0)}}=\frac{3.9}{1+\frac{5.04458 N}{M}}
$$

$a=a-\beta=3.9-\beta$

$$
A=\frac{C_{N}(0)}{E\left(\lambda_{N}\right)}+\frac{N x E\left(\lambda_{N}\right)}{M}=0.1+0.52 \frac{N}{M}
$$

The number of quantization levels $M$ is chosen arbitrarily, but a value $\mathrm{M}=20 \mathrm{xN}$ is large enough to cover all practical bit rates.

The process in Figure 2 can be decomposed into a superposition of simpler processes. The rate $\bar{\lambda}_{N}(t)$ can be thought of as the aggregate rate from $M$ independent identical ON - OFF minisources, each one being modeled as in Figure 3.

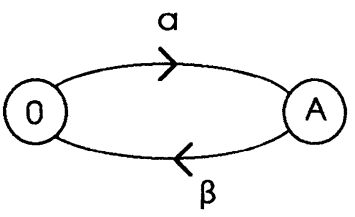

Figure 3: Minisource model

Each minisource alternates between transmitting 0 bits/pixel (OFF state) and $\mathrm{A}$ bits/pixel (ON state) according to a Bernoulli distribution. The transition from $O N$ to $O F F$ state occurs with rate $\beta$, while the transition from $O F F$ to $O N$ state occurs with rate $a$. The $\mathrm{ON}$ and $\mathrm{OFF}$ periods are exponentially distributed. 
The label in each state indicates the data rate of a minisource in that state. Thus, the state of the aggregated arrival process can be represented by the number of minisources, which are in the $\mathrm{ON}$ state. Assuming that $\mathrm{M}=20 \mathrm{xN}$, the values of the parameters $A, a, \beta$ and $p$ are given by:

$\mathrm{A}=0.126$ (bits/pixel) $\quad \mathrm{a}=\mathbf{0 . 7 8 5} \quad \beta=3.114 \quad \mathrm{p}=0.201$

The parameters of the model presented at [13] were chosen to match the experimental measurements. The experiment consisted of a 10s (300 frames) sequence of a video-telephone scene. The instantaneous bit-rate was measured in bits/pixel. Considering that there are about 250,000 pixels per frame and 30 frames per second, $1 \mathrm{bit} / \mathrm{pixel}$ corresponds to $7.5 \mathrm{Mbit} / \mathrm{s}$. Also, it was shown that each source has an average bit rate $\mathrm{E}(\lambda)=3.9 \mathrm{Mbit} / \mathrm{s}(0.52 \mathrm{bits} / \mathrm{pixel})$.

In our simulation, we consider that a time unit is $98.461 \mu \mathrm{sec}$ $\left(48 \times 8 / 3.9 \times 10^{6}\right)$, which corresponds to the packetizing delay for $3.9 \mathrm{Mbit} / \mathrm{s}$ video. Also the ATM cell size is 424 bits (48 information bytes and 5 overhead bytes). So, the maximum number of video cells per time unit carried on a SONET STS$3 \mathrm{C}(155.52 \mathrm{Mbit} / \mathrm{s})$ trunk is about equal to: $\mathrm{cvD}=35$ cells/time unit. $\left(c_{V D}=150 \times 10^{6} \times 98.461 \times 10^{-6} / 424\right)$.

Assuming that the channel speed $\mathrm{c}$ is equal to $155.52 \mathrm{Mbit} / \mathrm{s}$, the average load due to video traffic is $\rho=[\mathrm{E}(\lambda) \times \mathrm{N}] / \mathrm{c}$. The following table 1 shows the average load due to video traffic as a function of the number of $\mathrm{N}$ statistically independent video sources.

Table 1

Average load due to video traffic

$\mathrm{N} \mathrm{VD}$

Number of video sources $\rho$

Average load

$\begin{array}{cc}8 & 0.20 \\ 16 & 0.40 \\ 24 & 0.60 \\ 32 & 0.80\end{array}$

\subsection{TRAFFIC MODEL FOR VOICE SOURCES}

In voice traffic there is a strong correlation among cells arrivals from voice sources. The arrival process of voice calls is described by a Poisson process, while the durations of voice calls are described by an exponential distribution. The correlated generation of voice cells during a voice call is modelled by an Interrupted Poisson Process (IPP) [14]. Each voice source is described by an ON OFF model. During the ON state (talkspurts or active period) voice cells are 
generated according to a Bernoulli distribution, while during the OFF state (silent or inactive period) no cells are generated. The transition from $\mathrm{ON}$ to OFF state occurs with probability $\beta$ and the transition from OFF to ON state occurs with probability $a$. The $O N$ and OFF periods are geometrically distributed with mean $1 / \beta$ and $1 /$ a respectively (Figure 4 ).

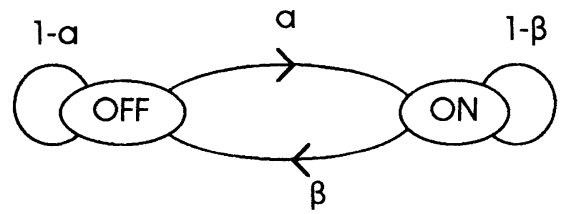

Figure 4: IPP model

The probability $P_{n}$ that $n$ out of $N$ voice sources are in the ON state is given by:

$P_{n}=\frac{N !}{n !(N-n) !}\left(\frac{a}{a+\beta}\right)^{n}\left(\frac{\beta}{a+\beta}\right)^{N-n}$

The state transition diagram for the number of active voice sources is shown in Fig.5.

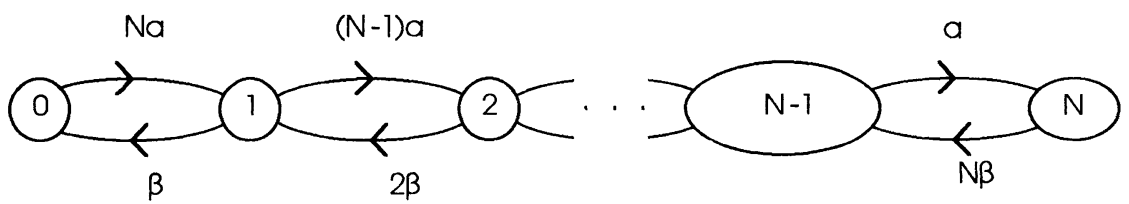

Figure 5: Model for the number of active voice sources

Voice traffic has the following parameters, which have also been used in the simulation model in [15]. One time unit is $6 \mathrm{~ms}$ and corresponds to the packetizing delay for $64 \mathrm{kbit} / \mathrm{sec}$ audio. The ATM cell size is $8 \times 53=424$ bits (48 information bytes and 5 header bytes). We consider a SONET STS-3C (155.52 $\mathrm{Mbit} / \mathrm{s}$ ) trunk, which carries a maximum of $\mathrm{cvo}_{\mathrm{v}}=2123$ cells per time unit $(150,000,000 \times 0.006 / 424)$.

The lengths of a silence period follow a geometrical distribution with mean $650 \mathrm{~ms}$ or $108.34(650 / 6)$ time units. The silence periods last $K_{I}$ time units, where $\mathrm{P}\left(\mathrm{K}_{\mathrm{I}}=\mathrm{k}\right)=\left(1-\mathrm{p}_{1}\right)^{\mathrm{k}-1} \mathrm{p}_{1}, \mathrm{k}=1,2, \ldots$ with $\mathrm{p}_{1}=6 / 650=0.0092$. During the silence period no cells are generated. The lengths of a talkspurt period follow a geometrical distribution with mean $352 \mathrm{~ms}$ or $58.67(352 / 6)$ time units. The talkspurt periods

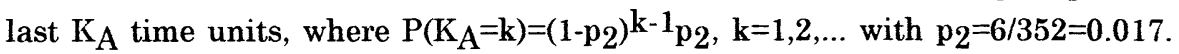
During the talkspurt period a voice call generates 1 cell per time unit. 
Considering $\mathrm{N}_{\mathrm{V}}$ independent voice calls, the steady state probability distribution of the number of active voice calls is binomial with probability $p$ (in the ON state) equal to $352 /(352+650)=0.3513$.

The following table 2 shows the average load due to voice traffic as a function of the number of voice sources.

Table 2

Average load due to voice traffic

\begin{tabular}{ccc}
\hline & & \\
Nvo $\left(\mathrm{Nvo}_{\mathrm{v}}\right)$ & $\rho=\mathrm{E}\left(\mathrm{N}_{\mathrm{vo}}\right) / \mathrm{c} v \mathrm{O}$ \\
$\begin{array}{c}\text { Number of voice } \\
\text { sources }\end{array}$ & $\begin{array}{c}\text { Average number of } \\
\text { active voice sources }\end{array}$ & \\
\hline & & \\
604 & 212 & 0.10 \\
1210 & 425 & 0.20 \\
1813 & 637 & 0.30 \\
2417 & 849 & 0.40 \\
\hline
\end{tabular}

\subsection{TRAFFIC MODEL FOR DATA SOURCES}

The generation of data from a data source is described by a Poisson arrival process. The probability $d(i)$ that $i$ data cells of data traffic arrive during each time slot, is given by:

$d(i)=\frac{\lambda_{i}^{d} e^{-\lambda} d}{i !}$

where $\lambda_{\mathbf{d}}$ is the mean arrival rate of data cells.

In this paper this traffic model for data sources is used for the simulation of the Loss Sensitive Class (critical data) and of one of the two Delay Sensitive Classes (regular real-time data with tolerance in loss).

\section{DESCRIPTION OF THE FCPS}

Since different traffic classes have different service requirements, a queue management algorithm is essential to discipline the queued cells. The buffered cells are served according to the FCPS, which manages dynamically the QoS requirements of each of the four classes. The FCPS has the following characteristics:

$i$. The FCPS is a dynamic priority method. Contrary to the fixed priority method it does not always assign a fixed high priority to one service class, but 
takes into account the delay time and/or the loss probability of the cells of each service class in each time-slot and makes an appropriate decision about the transmission of the buffered cells.

ii. The FCPS is applied on a multiple buffer structure rather than on a single buffer structure, because the latter appears difficulties in the preservation of the QoS requirements of each class.

iii. The FCPS makes provision for the loss and the delay time of the cells of each service class in each time-slot, considering the sensitivity (loss and/or delay) of each class. As it is shown in the simulation results, this characteristic ensures a low cell loss probability and acceptable limits of the cells' delay time.

$i v$. In the FCPS higher priority queues have nonpreemptive priority over lower priority queues.

$v$. The FCPS uses two priority methods: a space priority method and a service priority method. The space priority method is used for the control of the buffer space when an overflow condition (LDSC, LSC and DSC) occurs and/or when the expiration of the maximum admissible delay of the delay sensitive cells (LDSC and DSC) takes place. The service priority method is used as a scheduling method and assigns transmission priorities to the buffered cells.

In the following, these two priority methods are described in detail.

\section{A. Space priority method}

Upon the arrival of a cell of any of the four classes and if the corresponding queue is not full, then the cell is stored in it; otherwise it is discarded. Also, each arriving delay sensitive cell (video, voice and regular realtime data) has an associated laxity, which is reduced by one at each time-slot. The laxity of a delay sensitive cell is defined as the number of slots remaining before its deadline expires. A delay sensitive cell remains in the corresponding buffer until it is either transmitted or discarded (considered lost) when its laxity reaches zero.

\section{B. Service priority method}

A Queue Length Threshold (QLT) value is considered for each queue of the Loss and Delay Sensitive Class (LDSC) and of the Loss Sensitive Class (LSC). The proposed scheduling method (FCPS) takes into account the cell loss and delay requirements of each of the four classes and serves the buffered cells according to the following algorithm:

If the number of the buffered video cells is above a threshold value (QLT of LDSC buffer), then four video cells are transmitted to the four output links. Note that, the number of the transmitted cells is equal to the number of output links. Otherwise, priority is given to the critical data cells; if the number of the buffered critical data cells is above a threshold value (QLT of LSC buffer), then four critical data cells are transmitted to the output links. Since the video cells are 
loss sensitive and delay sensitive, the FCPS takes into account both these characteristics. So, if the number of the buffered video cells and the number of the critical data cells are below the corresponding threshold values, then the video queue, the voice queue and regular real-time data queue are searched, in order to find the cell(s) with the minimum laxity in each queue. The cells with the minimum laxity are those that have been remained in the corresponding buffers for the longest time. In this case the minimum laxities are compared with each other and four cells with the smallest laxities of the minimum laxities are transmitted. This feature stamps the proposed FCPS as a high flexibility dynamic priority scheme. However, if there are delay sensitive cells with equal minimum laxities, then priority is given first to the video cells, next to the voice cells and last to the regular real-time data cells. For example, if there are 1 video cell, 2 voice cells and 3 regular real-time data cells with equal minimum laxities, then 1 video cell, 2 voice cells and 1 regular real-time data cell will be transmitted to the four output links.

Each class buffer has a FIFO (First In First Out) structure, controlled by its control logic, which informs the arbiter about the current queue length and the delay time of the delay sensitive buffered cells. This structure guarantees that the cells' sequence of each class is preserved as they are transmitted to the output links. This is true even in the case that the arbiter decides to transmit the cells with the smallest laxities of the minimum laxities among the buffered cells in the three delay sensitive classes. The delay sensitive cells of a class are stored in the corresponding buffer in successive locations. The first buffer locations are occupied by cells with laxity equal to the Minimum Laxity (ML), the next locations by cells with laxity equal to ML-1 and so on. According to the FCPS, when the arbiter decides to extract a cell of a delay sensitive class (this one with the minimum laxity) from the corresponding buffer for transmission, then the cell in the first buffer location is transmitted and the cells in the next locations are shifted one location ahead their current location. A similar operation takes place in the case that the arbiter decides to transmit more than one cells of a delay sensitive class in a given time-slot. Also, each control logic of a delay sensitive class buffer uses a counter, which starts with a value equal to the corresponding deadline and is reduced by one at each time slot. When it reaches zero, it informs the arbiter about this state. In this case, if the arbiter decides that no delay sensitive cell has to be transmitted in the current time slot and there are some delay sensitive cells, say $w$, that have not been transmitted yet and their laxity has reached zero, then the cells in the delay sensitive buffer are shifted for $w$ locations ahead of their current locations. In this way the delay sensitive cells that have not been transmitted yet and their laxity has reached zero, are destroyed.

In the proposed priority scheme, the number of the video cells and the number of the critical data cells have to be calculated in each time-slot, in order to be compared with the corresponding QLT value. Also, in a given time-slot the minimum laxities of the cells in the video, voice and regular real-time data buffers have to be compared with each other in order to find the four cells with the smallest laxities of the minimum laxities. All the above calculations seem to 
involve heavy processing in each time-slot. So, a fast and flexible algorithm is needed for the implementation of the proposed FCPS. Although several implementation schemes for various priority methods have been presented in the literature [16] - [20], a special more approaching possible solution can be found in [21]. In that paper three ATM service classes were considered (LDSC, LSC and DSC), which were served according to a priority scheme applied to the output buffers of an ATM switch. A shared output buffer was used for each class and a special algorithm was applied in order to calculate the number of the stored cells in each buffer and the number and the laxities of the buffered delay sensitive cells.

\section{SIMULATION MODEL}

In this section, we describe the simulation model for evaluating the proposed FCPS. Four service classes are considered and the cells of each class are generated according to the models described in section 3 . The loss and/or delay sensitivity of each class is shown in Table 3.

Table 3

Traffic sensitivity

\begin{tabular}{ccc}
\hline Service Class & Loss Sensitivity & Delay Sensitivity \\
\hline Video & Yes & Yes \\
Critical Data & Yes & No \\
Voice & No & Yes \\
Regular Real-Time & No & Yes \\
Data & & \\
\hline
\end{tabular}

As it is shown in Table 3, video cells are sensitive to both cell loss and delay time, critical data cells are loss sensitive and have not strict requirements of cell delay variation, while the voice cells and the regular real-time data cells have strict and different delay requirements but they can tolerate some losses. The transfer bit rate is assumed to be $150 \mathrm{Mbit} / \mathrm{s}$. The maximum end-to-end delays of video, voice, critical data and regular real-time data cells are assumed to be $10 \mathrm{~ms}, 10 \mathrm{~ms}, 100 \mathrm{~ms}$ and $20 \mathrm{~ms}$ respectively. If the number of intermediate ATM nodes is assumed to be equal to 10, then the delay times of video, voice, critical data and regular real-time data cells in the MIMO multiplexer become $1 \mathrm{~ms}, 1 \mathrm{~ms}, 10 \mathrm{~ms}$ and $2 \mathrm{~ms}$ respectively. These delay times correspond to 360,360 , 3600 and 720 time-slots respectively. Table 4 shows the loss and delay requirements for the four ATM service classes: 
Table 4

Cell loss and delay requirements of each traffic type

\begin{tabular}{ccc}
\hline Service Class & Loss & Delay per ATM node \\
\hline Video & $10^{-9} \sim 10^{-12}$ & $1 \mathrm{~ms}$ (360 time-slots) \\
Critical Data & $10^{-6} \sim 10^{-9}$ & $10 \mathrm{~ms}$ (3600 time-slots) \\
Voice & $<10^{-3}$ & $1 \mathrm{~ms}(360$ time-slots $)$ \\
Regular Real-Time \\
Data
\end{tabular}

The simulation for the various combinations of traffic loads of the four service classes leads to a large number of simulation runs. In order to decrease the number of simulation runs, we ran our simulation for two fixed values of video load, $20 \%$ and $60 \%$. Table 5 shows the load assignment for $100 \%$ total load for the two cases of the video load:

Table 5

Load assignment for $100 \%$ total load

Service Class

Load

\begin{tabular}{ccc}
\hline Video & $20 \%$ & $60 \%$ \\
Critical Data & $30 \%$ & $10 \%$ \\
Voice & $30 \%$ & $20 \%$ \\
Regular Real-Time & $20 \%$ & $10 \%$ \\
Data & & \\
\hline
\end{tabular}

The relation among the loads of the four service classes is valid for every value of the total offered load. For example, if the total offered load is $80 \%$ and the fixed video load is $20 \%(60 \%)$, then the critical data load is $22.5 \%(5 \%)$, the voice load is $22.5 \%(10 \%)$ and the regular real-time data load is $15 \%(5 \%)$.

Finally, we considered two cases of the buffer sizes with different Queue Length Thresholds for each case in the video and in the critical data buffer. Table 6 shows the buffer sizes and the corresponding values of QLT for each case. The numbers without parenthesis correspond to the buffer sizes, while the 
numbers inside the parenthesis correspond to the values of QLT for each case in the video and in the critical data buffer.

Table 6

Buffer sizes and corresponding QLT values

Service Class

Buffer size (QLT)

\begin{tabular}{cccc} 
Video & $48(30)$ & $48(40)$ & $64(56)$ \\
Critical Data & $48(30)$ & $48(40)$ & $64(56)$ \\
Voice & 48 & 48 & 64 \\
$\begin{array}{c}\text { Regular Real-Time } \\
\text { Data }\end{array}$ & 48 & 48 & 64 \\
\hline
\end{tabular}

\section{SIMULATION RESULTS}

In this section, some results concerning the loss probability and the mean delay of the cells of each service class are presented for three priority control methods: No priority (First Come First Serve), Fixed priority and Four Classes Priority Scheme. The simulation time is $10^{7}$ time-slots.

Figures 6 and 7 show the voice and the real-time data cell loss probabilities as a function of the total offered load, for fixed video loads equal to $20 \%$ and $60 \%$ respectively. Figures 8 and 9 show the video and the critical data cell loss probabilities as a function of the total offered load, for fixed video loads equal to $20 \%$ and $60 \%$ respectively. In these four figures the buffer size is equal to 48 and the QLT in the video and the critical data buffer is equal to 40 .

In the case where no priority control is applied (FCFS), the voice and the real-time data loss probabilities are inside acceptable limits, while the video and critical data losses become seriously high, because the four classes are not served according to their QoS requirements. Note that, for the simulation of the no priority control method a common buffer for all classes was used with capacity equal to $4 \times 48=192$ cells.

When the fixed priority control is used, the highest priority class cells are transmitted first, while the lower priority class cells are transmitted only in the case where the higher priority buffer is empty. The priority order is first the video cells, next the critical data cells, next the voice cells and last the real-time data cells. In this case, video cells are not lost for all cases of traffic load and the critical data cells are lost when the video traffic is equal to $60 \%$. Voice cells and real-time data cells are seriously affected by higher priority classes and present high losses.

When the Four Classes Priority Scheme is applied, the loss probabilities of all classes of traffic are improved significantly and are inside the acceptable 
limits for a large range of traffic load. When the video traffic load is equal to $60 \%$, the loss probabilities of the voice traffic and of the real-time data traffic are not affected so much as in the fixed priority method. However, the loss probability of the voice traffic is greater than the loss probability of the real-time data traffic for all cases of traffic load, because of the smaller deadline of the voice cells and the higher load of the voice traffic compared with the respective values of the real-time data traffic. The video traffic appears loss probability greater than $10^{-7}$, when the total offered load is above $90 \%$, for fixed video load $60 \%$. This low loss probability of the video traffic is due to the proposed FCPS, which takes into account the loss and the delay sensitivity of the video cells and serves four cells in each time-slot.

Figure 10 shows the loss probability of three service classes (critical data, voice and real-time data) as a function of the total offered load, for fixed video load equal to $60 \%$, under the application of the proposed FCPS. The buffer size is equal to 48, while two cases of the QLT in the video and the critical data buffer are considered: 40 and 30. The loss probabilities of the video traffic are not shown, because they are smaller than $10^{-7}$, except for the case where the video load is equal to $60 \%$, the buffer size is 48 and the QLT is equal to 40 . The loss probabilities of the video traffic for these values of parameters are shown in Fig. 9. When $\mathrm{QLT}=30$, the loss probabilities of the voice traffic and the real-time data traffic are higher than the respective values, in the case where the $Q L T=40$. By contrast with the voice traffic and the real-time data traffic, the loss sensitive cells (video and critical data) present lower loss probabilities, in the case where QLT=30. This is due to the following: As the QLT decreases from 40 to 30 , the number of the buffered loss sensitive cells reaches more often the QLT value and transmission priority is given to the loss sensitive cells. That involves lower losses for the video and critical data classes, but higher losses and delays for the voice and real-time data classes.

Figure 11 shows the loss probability of three service classes (critical data, voice and real-time data) as a function of the total offered load, for video load equal to $60 \%$, under the application of the proposed FCPS. Two cases of buffer size are considered: 48 and 64 with QLT values in the video and the critical data buffer 40 and 56 respectively. The loss probabilities of the video traffic are not shown, because they are smaller than $10^{-7}$. We can observe that the greater buffer size results to lower loss probabilities for all classes. However, as the buffer size increases from 48 to 64 , the loss sensitive cells remain for longer time in the corresponding buffer and present greater mean delays.

Figures 12 and 13 show the mean buffer delay of the video, critical data, voice and regular real-time data cells as a function of the total offered load, for two cases of video load, $20 \%$ and $60 \%$, under the application of the proposed FCPS. We plotted two graphs in order to distinguish the lines between the curves. The buffer size is equal to 48 and the QLT value in the video and the critical data buffer is 40 . We observe that even for high loads, the delay specifications of Table 4 are satisfied. When the video load increases from $20 \%$ to $60 \%$, the mean delays of the critical data, voice and real-time data cells become greater, while the mean delay of the video cells decreases. This is due to the FCPS, which assigns higher priority to the video cells. The mean cell delay of the 
real-time data class is greater than that of the voice class, because the latter has smaller deadline (more strict delay requirements).

Figure 14 shows the loss probability of the buffered voice and real-time data cells, due to the expiration of their laxity as a function of the total offered load, for video load $20 \%$ and $60 \%$. The buffer size is equal to 48 and the QLT in the video and the critical data buffer is equal to 40. The loss probability is greater for low traffic loads of these two classes, because FCPS assigns higher priority to the high load $(60 \%)$ of video traffic. Even for high total offered loads, the cell loss probabilities of the voice and real-time data classes are lower than acceptable limits.

\section{CONCLUSIONS AND FURTHER WORK}

In this paper we have proposed a new dynamic priority control scheme, called Four Classes Priority Scheme. Its performance has been studied by simulation under its application on a 16x4 MIMO multiplexer, where four ATM service classes were considered with specific QoS requirements. The results have shown that this scheme satisfies efficiently the QoS requirements of each class for a large range of traffic load.

Further research work may include a more detailed investigation on various parameters, such as the buffer length of each output queue, the value of the Queue Length Threshold in the LDSC and the LSC and the load of each service class as a function of the total offered load.

\section{REFERENCES}

1. CCITT: I-Series Recommendation on B-ISDN. Geneva, 1991.

2. G. Galassi. G.Rigolio and L.Verri: Resource Management and Dimensioning in ATM Networks. IEEE Network Magazine, Vol. 4, No. 3, May 1990, pp. 8-17.

3. R. Steinmetz and T. Meyer: Modelling Distributed Multimedia Applications. Proceedings IWACA 92, Munich, pp.337-349.

4. T. Akaike, T. Takenaka, and K. Takami: Evaluations of multimedia packet switching system with bursty sources. IEICE Technical Report, SE 87-42, pp. 1-6, 1987.

5. R.Chipalkatti, J.F.Kurose, D.Towsley: Scheduling policies for real-time and nonreal-time traffic in a statistical multiplexer. Proceedings IEEE INFOCOM '89, pp.774-783.

6. Y.Lin, J.Kobza: Analysis of a delay-dependent priority discipline in a multiclass traffic packet switching node. Proceedings IEEE INFOCOM ' 88 , pp.9A.4.1-9A.4.1.10.

7. D.Ferrari, D.C.Verma: A scheme for real-time channel establishment in wide-area networks. IEEE J. Sel. Areas Commun. Vol.8, No.3, April 1990, pp. 368-379.

8. S.Pingali, J.F.Kurose: On scheduling two classes of real-time traffic with identical deadlines. Proceedings of IEEE Globecom'91, Phoenix AZ, 1991, pp.460-465. 
9. P. Ganos, M. Koukias, S. Kotsopoulos and G. Kokkinakis: A new dynamic priority queueing scheme at an ATM switch. First UK Workshop on performance modelling and evaluation of ATM networks, University of Bradford, Bradford, UK, Jun. 1993

10. M.W.Beckner, T.T.Lee, and S.E.Minzer: A protocol and prototype for broadband subscriber access to ISDN. Proceedings ISS 87, Phoenix, AZ, Mar. 1987.

11. A. Pattavina: Multichannel bandwidth allocation in a broadband packet switch. IEEE J. Select. Areas Commun. Vol. 6, No. 9, Dec.1988, pp.14891499.

12. M.J.Karol, M.G.Hluchyj, S.P.Morgan: Input versus output queueing on a space-division packet switch. IEEE Trans. Commun. Vol.35, No.12, , Dec.1987, pp.1347-1356.

13. B.Maglaris, D.Anastassiou, P.Sen, G.Karlsson, J.Robins: Performance analysis of statistical multiplexing for packet video sources. Proceedings IEEE Globecom'87, Tokyo, Nov.1987, pp.1890-1899.

14. J.N.Daigle, J.D.Langford: Models for analysis of packet voice communications systems. IEEE J. Select. Areas Commun Vol.SAC-4, Sept.1986, pp.847-855.

15. A.D.Gelman, S.Halfin, W.Willinger: On buffer requirements for store-andforward video on demand service circuits. Proceedings IEEE GLOBECOM '91, Phoenix AZ, 1991, pp.976-980.

16. C.B.Woodworth, M.J.Karol, R.D.Gitlin: A flexible broadband packet switch for a multimedia integrated network. Proceedings IEEE ICC '91, Denver, June 1991, pp.78-85.

17. W.T.Chen, H.J.Liu, Y.T.Tsay: A high-throughput cell scheduling algorithm for delay-sensitive traffic in broadband switching systems. Proceedings IEEE INFOCOM '92, May 1992, pp.2499-2508.

18. C.Oh, M.Murata, H.Miyahara: Priority control for ATM switching systems. IEICE Transactions on Communications, vol. E75-B, no.9, Sept. 1992, pp.894-905.

19. Y.Takagi, S.Hino, T.Takahashi: Priority assignment control of ATM line buffers with multiple QoS classes. IEEE J. Select. Areas Commun Vol.SAC-9, no.7, Sept.1991, pp.1078-1091.

20. H.J.Chao: A novel architecture for queue management in the ATM network. IEEE J. Select. Areas Commun Vol.SAC-9, no.7, Sept.1991, pp.1110-1118.

21. P.Ganos, M. Koukias, G. Kokkinakis: ATM switch with multimedia traffic priority control. Submitted for publication to European Transactions on Telecommunications and related technologies. 


\section{$\begin{array}{lll}\text { (1) Four Classes Priority Scheme (2) Fixed Priority } & \text { (3) No Priority }\end{array}$}

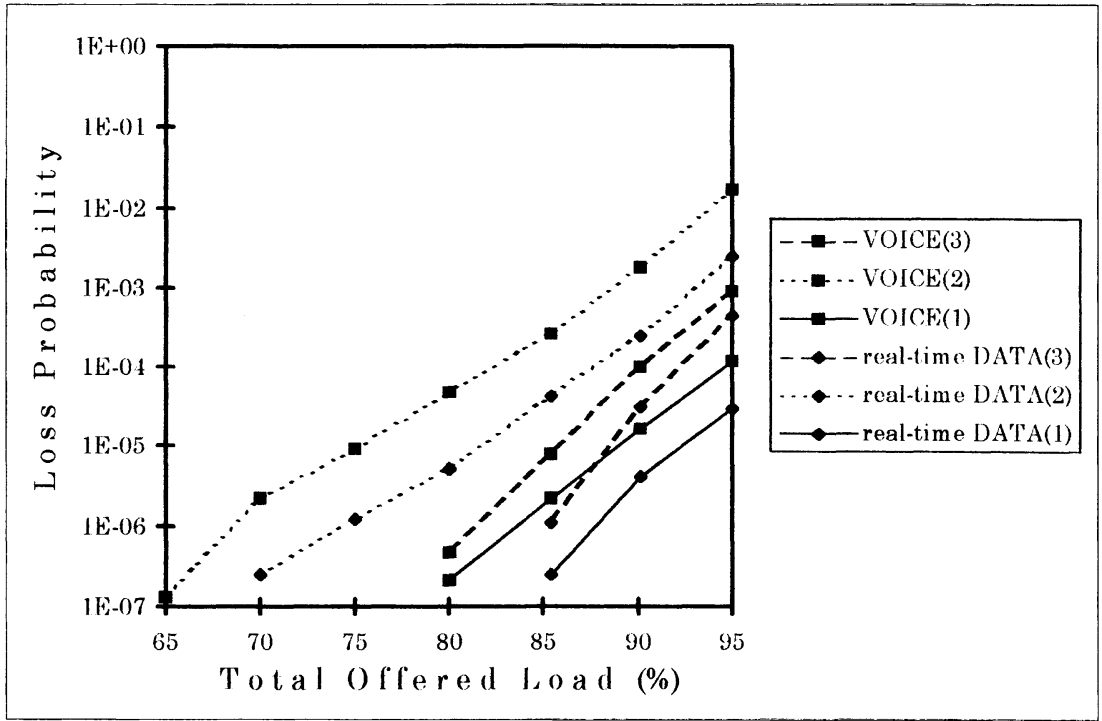

Figure 6: Loss probability versus total offered load for $20 \%$ video load, for buffer size $=48$ and $\mathrm{QLT}=40$.

\section{\begin{tabular}{|lll|}
\hline (1) Four Classes Priority Scheme & (2) Fixed Priority & (3) No Priority \\
\hline
\end{tabular}}

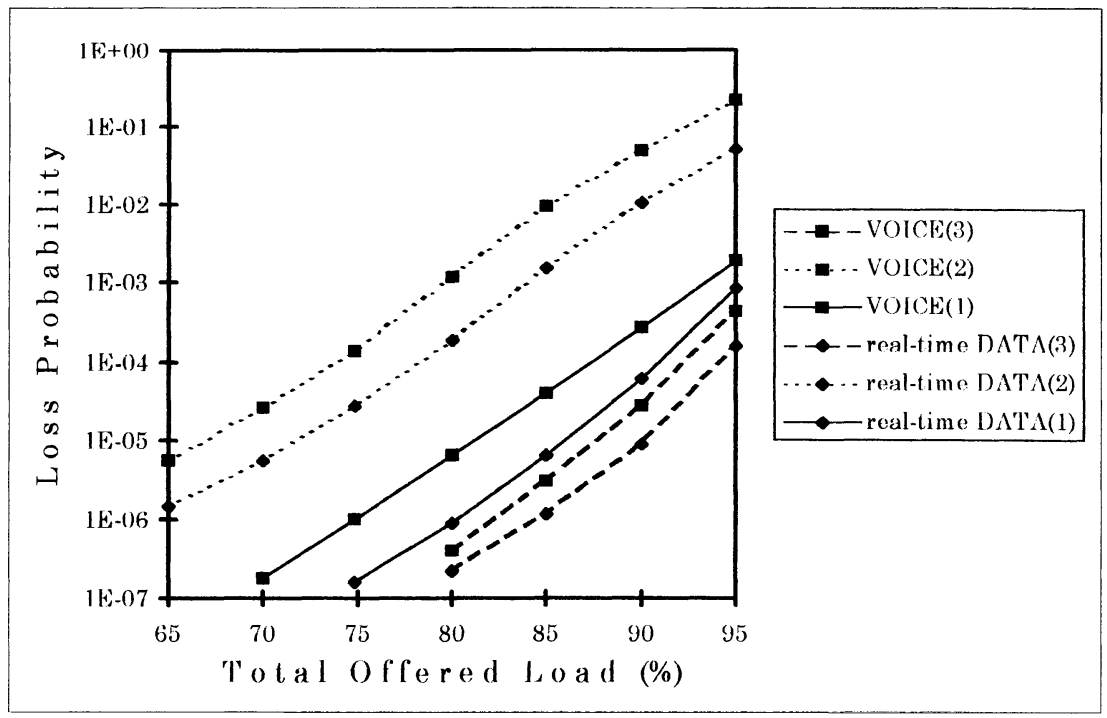

Figure 7: Loss probability versus total offered load for $60 \%$ video load, for buffer size $=48$ and QLT $=40$. 


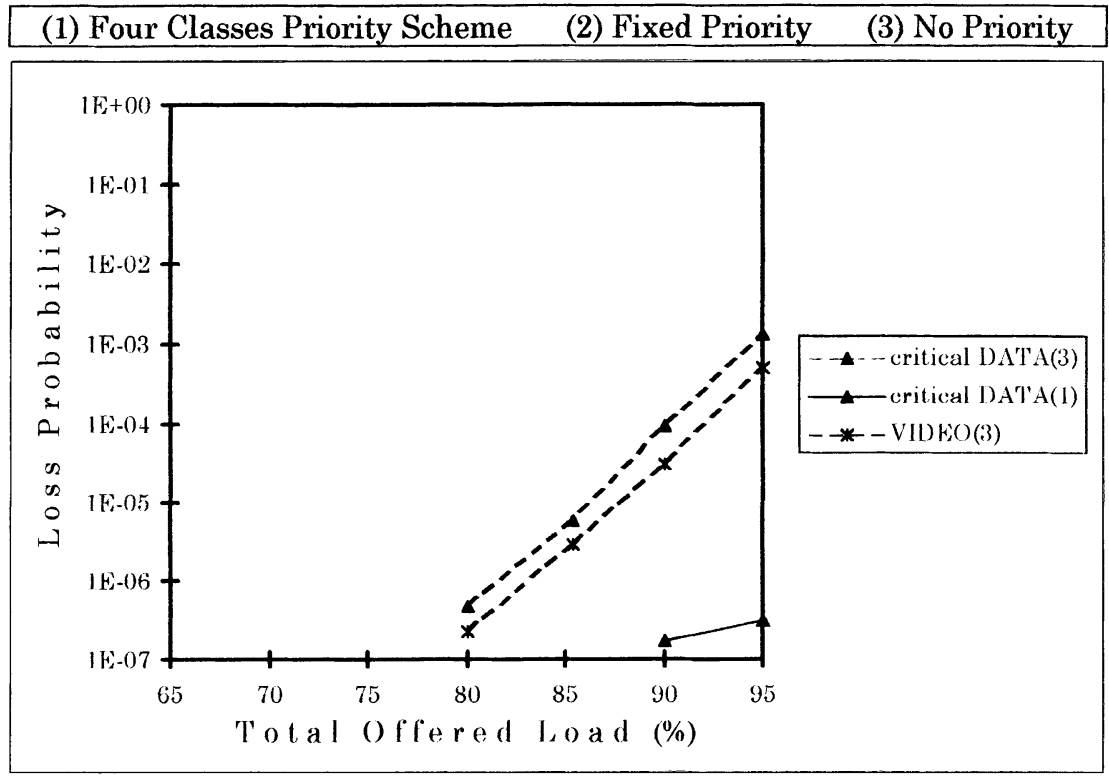

Figure 8: Loss probability versus total offered load for $20 \%$ video load, for buffer size $=48$ and $\mathrm{QLT}=40$.

\section{(1) Four Classes Priority Scheme (2) Fixed Priority $\quad$ (3) No Priority}

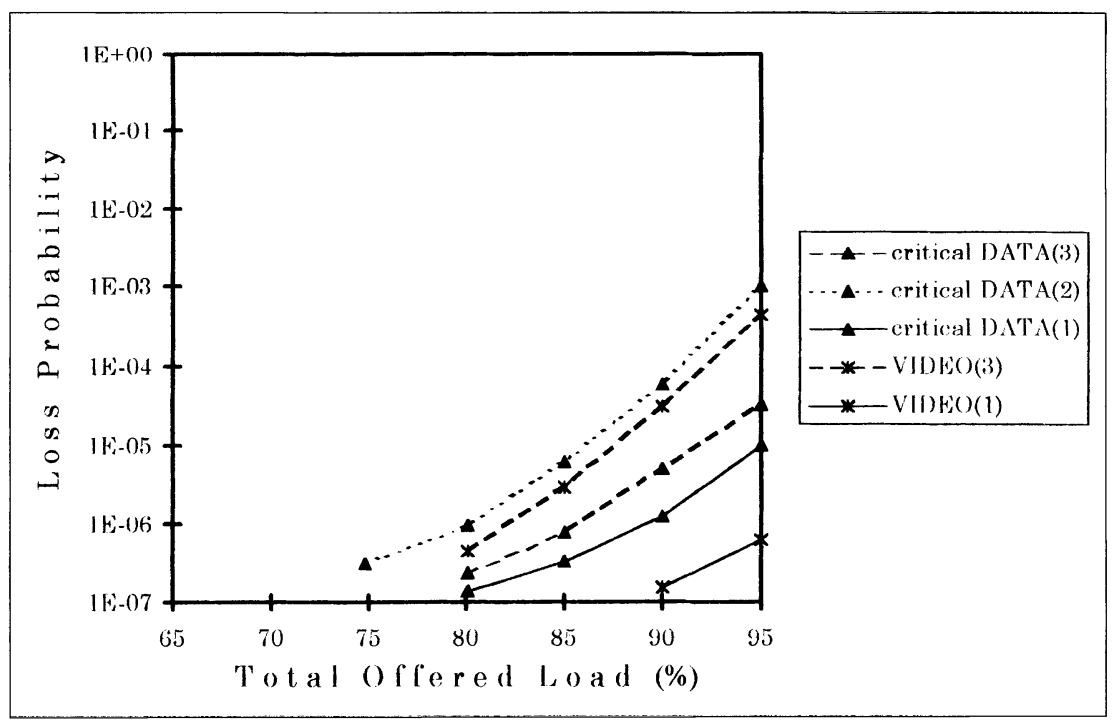

Figure 9: Loss probability versus total offered load for $60 \%$ video load, for buffer size $=48$ and $\mathrm{QLT}=40$. 


\section{$\begin{array}{ll}\text { (1) } \text { Buffer size }=48 \text { QLT }=40 & \text { (2) Buffer size }=48 \text { QLT }=30\end{array}$}

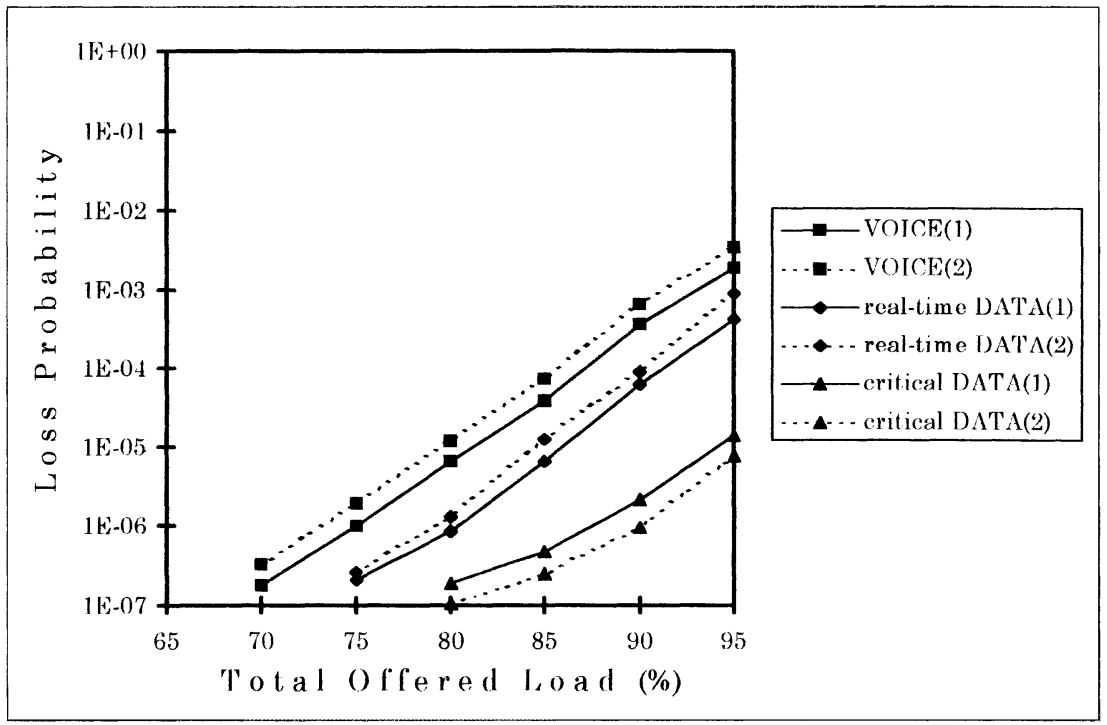

Figure 10: Loss probability versus total offered load for $60 \%$ video load, under the application of the Four Classes Priority Scheme.

\section{(1) Buffer size $=48$ QLT $=40$}

(2) Buffer size $=64$ QLT $=56$

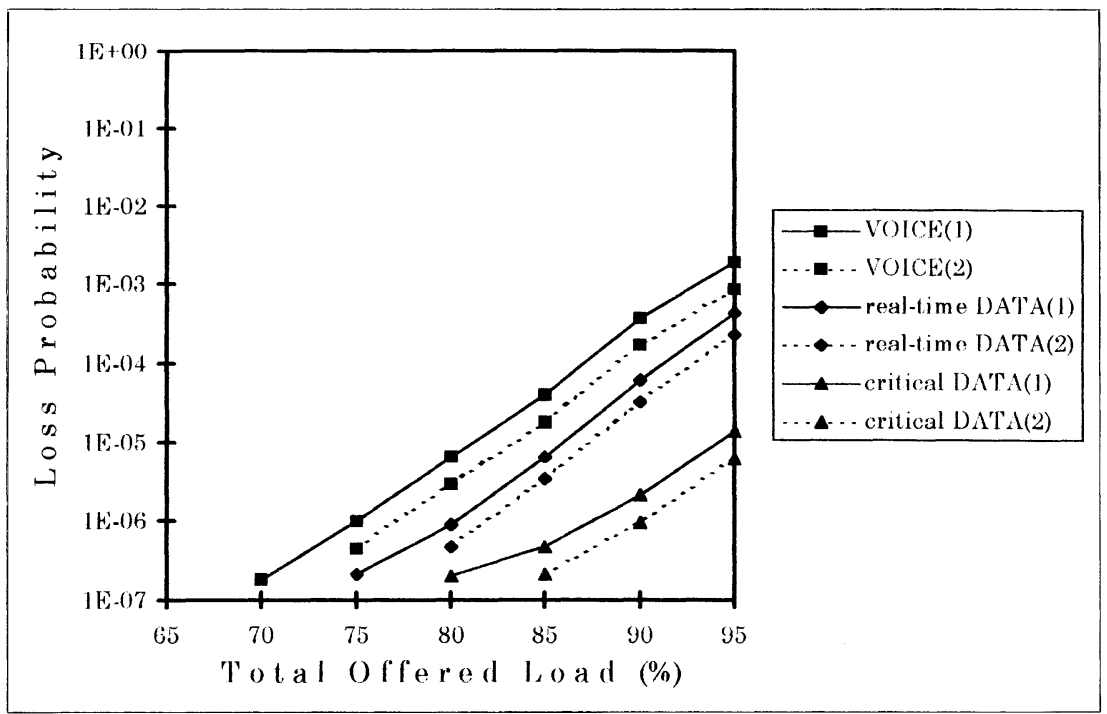

Figure 11: Loss probability versus total offered load for $60 \%$ video load, under the application of the Four Classes Priority Scheme. 


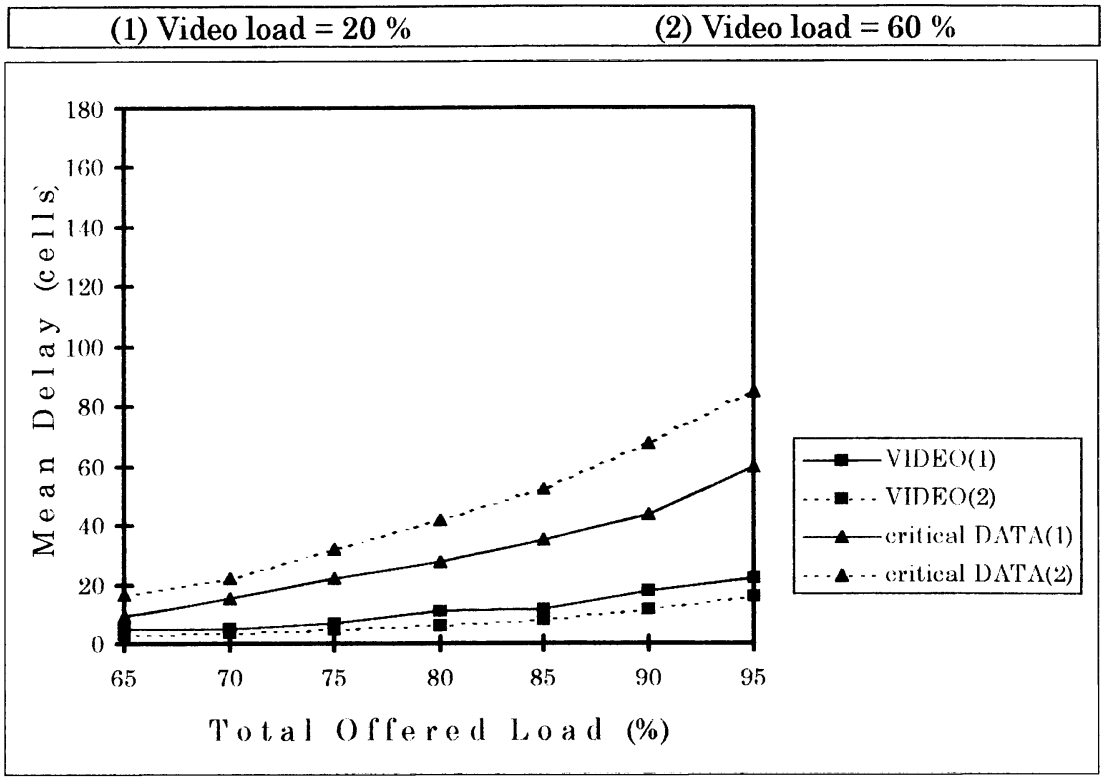

Figure 12: $\quad$ Mean delay (cells) versus total offered load for buffer size $=48$ and QLT=40 under the application of the FCPS.
(1) Video load $=20 \%$
(2) Video load $=60 \%$

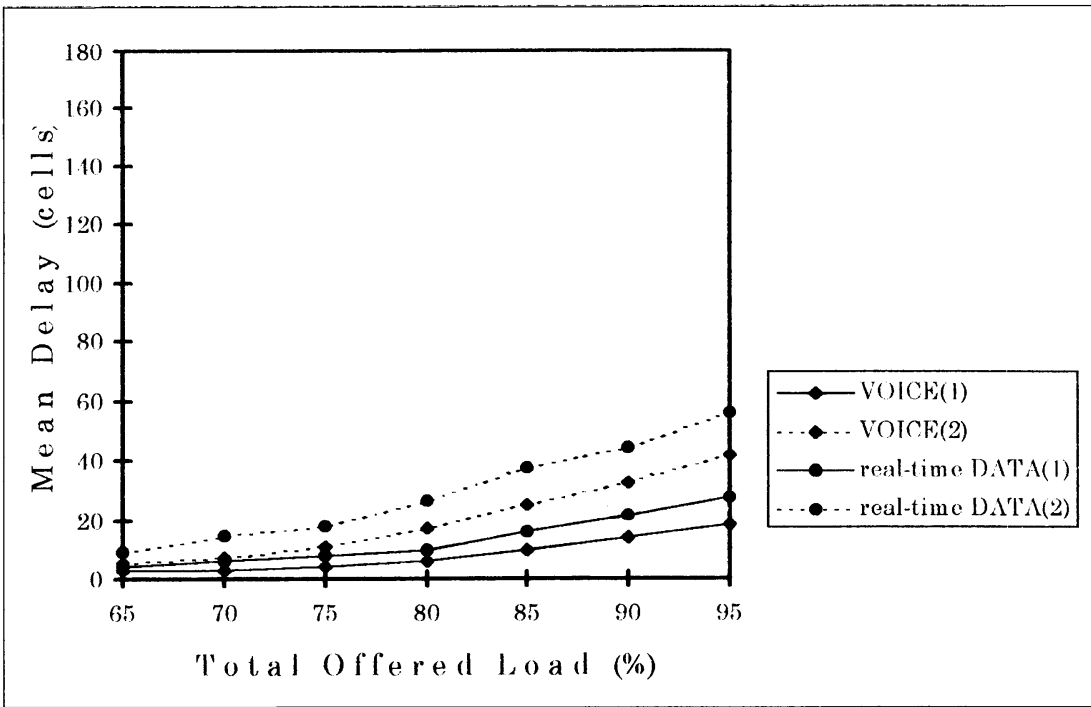

Figure 13: $\quad$ Mean delay (cells) versus total offered load for buffer size $=48$ and QLT=40 under the application of the FCPS. 
(1) Video load $=20 \%$

(2) Video load $=60 \%$

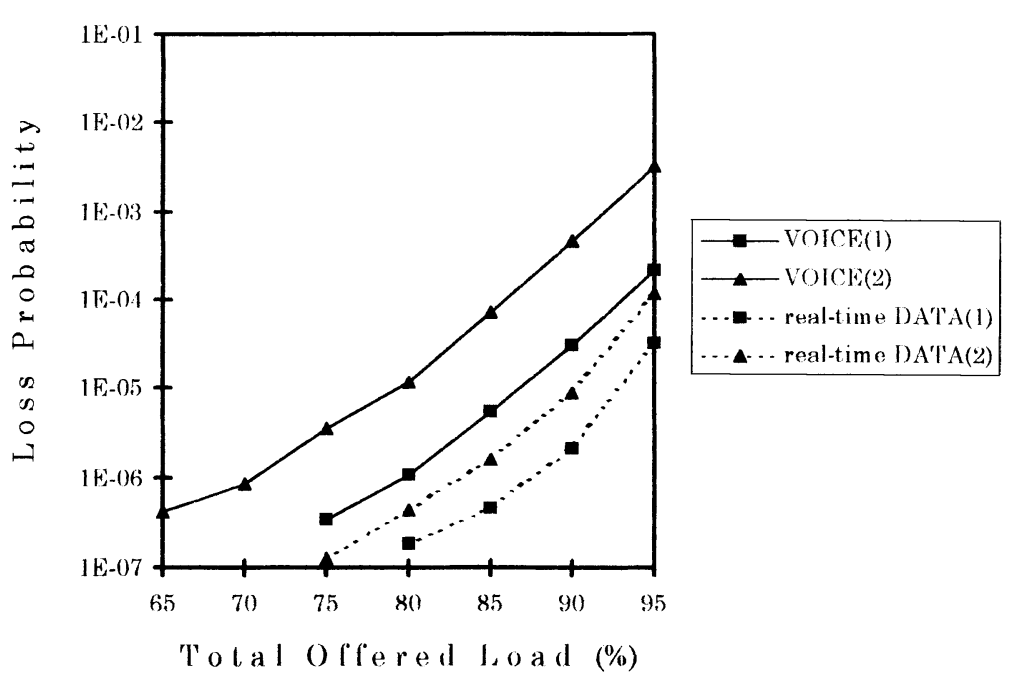

Figure 14: Loss probability due to laxity expiration versus total offered load for buffer size $=48$ and QLT=40 under the application of the FCPS. 